\title{
Paradigmatic Orientations of Mind among Physicists
}

\author{
Herman J. Pietersen \\ Professor, University of Limpopo, Turfloop Campus 0727, Republic of South Africa \\ herman.pietersen@ul.ac.za
}

\section{Doi:10.5901/mjss.2014.v5n23p2110}

\begin{abstract}
A meta-theory was developed that brought together implicit premises or world views that constantly re-surface in human thought. It consists of four paradigmatic or root intellectual orientations, designated as type I, type II, type III and type IV respectively. The theory was found to be applicable across a wide range of thinkers, scholarly disciplines, and cultures. In the current paper the framework is presented in terms of its main components and dynamics. The aim of the paper is to apply the meta-philosophical framework in identifying unique but complementary intellectual predispositions or modalities of mind among physicists. For this purpose a brief sketch of some prominent figures in physics is provided, showing their different orientations of mind. David Bohm represents the type I (metaphysical or objectivist-empyrean) tendency; Albert Einstein, the quintessential type II (scientific or objectivist-empiricist) approach; Carl Sagan is primarily the type III (subjectivist-empiricist) figure; whilst Fritjof Capra is the type IV (reforming or subjectivist-empyrean) representative in this group.
\end{abstract}

Keywords: paradigmatic modalities of mind, Bohm, Einstein, Sagan, Capra

\section{Introduction}

It is probably safe to say that in twentieth century science, as well as in the mind of the informed public, physics served and continues to serve as the ultimate reference point of exactitude in knowledge, and of what the human intellect is capable of achieving.

From the epoch-making formulation of fundamental, but mechanical, laws of nature by Isaac Newton, to the current sophisticated and dynamic conception of the universe as a complex and self-organizing whole; physics has captured the imagination of expert and layperson alike.

A central and dividing issue for physicists, which by its very nature invites philosophical consideration (see also Auyang, 1999, and Barrett, 2000), concerns the question of the 'true' nature of physical reality. Can one, in the realm of physics still speak of reality in a Cartesian sense as the world-out-there (res extensa), measurable by (albeit increasingly sophisticated and non-intuitive) extensions of the human sensory apparatus, or are the mathematical solutions of quantum theory the best way of conceiving physical reality? What is the solution to the conflict presented by a determinate physics (relativity theory) in which God does not play dice with the world (Einstein) and an indeterminate physics based on quantum theoretical logic in which the idea of a Universe by Design is discarded, and God is dropped from the Newtonian equation (Hawking); a universe in which the separation between observer and observed no longer makes sense in physics (Heisenberg)?

These issues seem to be still unresolved, as reflected by an anecdote reporting that the Nobel laureate, Murray Gell-Mann: "...spent years saying quarks should be understood as mathematical constructs, not actual things - then the particles were actually discovered, confounding those who had predicted them" (Easterbrook, 2000: 110).

Another Nobel laureate, the physicist, Steven Weinberg, writes that in natural science (including physics) there is not only no clear, a priori, conception of what the so-called "scientific method" is, but: " ...the working philosophy of most scientists is that there is an objective reality..." (Weinberg, 1995: 14). It would seem then that, on balance and as regulating principle for working scientists, there is indeed a reality independent of the observer, as Einstein had it (Barnett, 2000; Stenger, 1993), and that sub-atomic phenomena such as electrons, quarks and photons do exist.

The present paper is not about an intra-scientific discussion of physics as such, but an attempt to apply a metaphilosophical framework in identifying unique but complementary intellectual predispositions or modalities of mind among physicists. A fourfold scheme of basic knowledge paradigms is presented in the next section, followed by its use in a consideration of the approaches of a number of prominent figures in physics. 


\section{Fundamental Predispositions in Human Thought}

The meta-theory to be utilized here was developed in detail in a first volume (Pietersen, 2011) and continued with new applications in a second volume, (Pietersen, 2014) and will therefore only briefly be discussed.

Figure 1 shows four orientations in human thought that are the result of a combination of two axes (epistemological and ontological), namely: objectivist-empyrean (type I); objectivist-empiricist (type II); subjectivist-empiricist (type III), and the subjectivist-empyrean (type IV).

This is followed in Figures 2, 3, 4 and 5 by a description of characteristic elements of each of the four archetypes. These should be more or less self-explanatory, but it may be helpful to point out that each of the primary orientations address a different and basic question, namely: (a) what is this? (Type II); (b) what is behind this? (Type I); (c) what is wrong / wonderful about this? what is the story? (Type III); (d) what should be done about this? (Type IV).

Figure 1: The four orientations in human thought

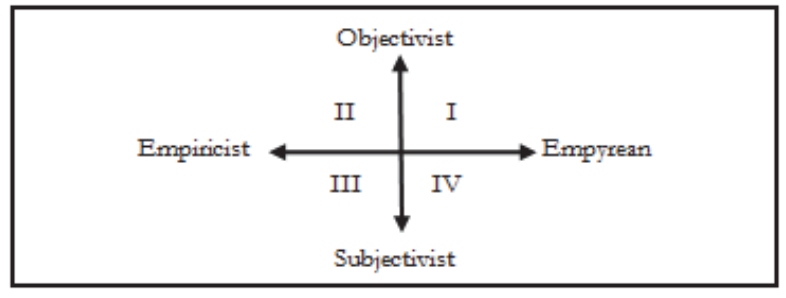

Figure 2: Objectivist-empyrean (type I) elements

Emphasize reason (rationality)
Impersonal
Theoretical/integrative
Macroscopic focus
Comprehensive understanding
Concerned with possible ideas

Figure 3: Objectivist-empiricist (type II) elements

\begin{tabular}{ll|} 
Emphasize reason (rationality) \\
Impersonal \\
Systematic-analytic \\
Microscopic focus \\
Detailed explanation \\
Concerned with verifiable ideas \\
\hline
\end{tabular}

Figure 4: Subjectivist-empiricist (type III) elements

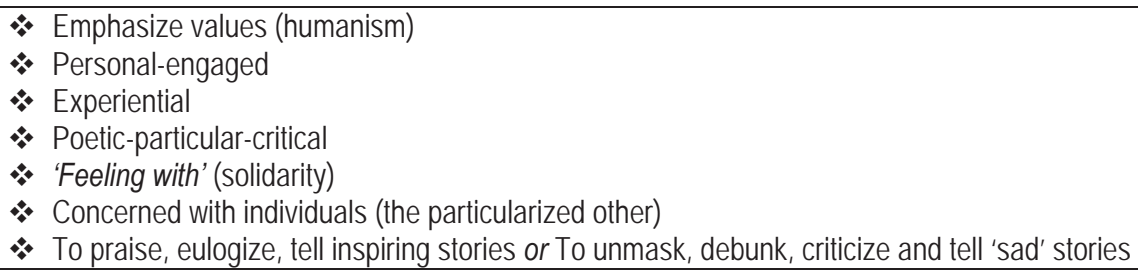


Figure 5: Subjectivist-empyrean (type IV) elements

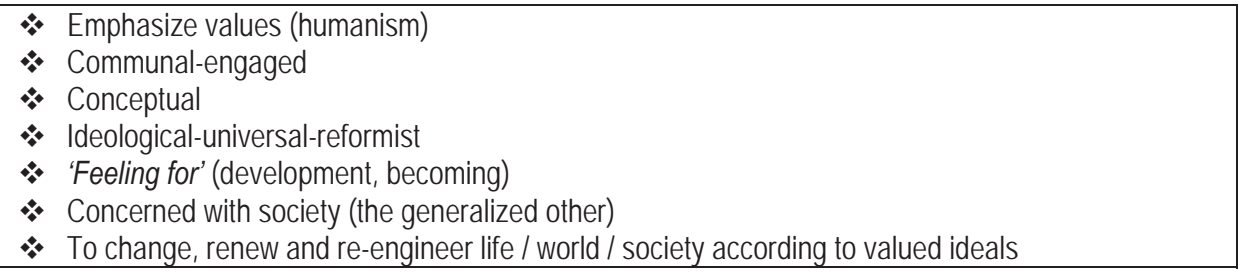

\subsection{The main principles}

The main propositions or theses of the meta-theory are as follows (Pietersen, 2011):

h) Fundamental orientations of mind predetermine the type of ideas, theories, "ways" of making sense of and dealing with the world.

i) Each orientation or modality of mind co-exists with other modes in a dynamic mutuality of conflict and complementariness.

j) Thought products (individually or collectively) reflect different mixes of the basic orientations, although dominant (primary) tendencies prevail as a result of root "preferences". No thinker functions entirely within a single (primary) mode of thought, but also in varying degrees interfaces with directly adjacent (secondary) modes. Tertiary orientations also manifest in each intellectual product, either in a conflicting, suppressed or underrepresented manner.

k) Together, the different modes of thought form a unity or whole with each giving meaning to the other and with each dependent on the existence of its opposite mode.

I) Attempted dominance by one modality of mind inevitably leads to countervailing reactions in other realms of thought. Adherents of highly divergent (directly opposing) orientations will more likely experience mutual misunderstanding, disaffection and even potentially irreconcilable beliefs and convictions.

$\mathrm{m}$ ) Knowledge endeavours in the world at large, in different cultures, disciplines and traditions of thought, and at different levels of analysis, show similarity in terms of underlying modalities of mind.

n) The limitations of one modality of mind are complemented by the strengths of others, especially its diagonally opposite mode.

\section{Some Paradigmatic Exemplars in Physics}

Before briefly highlighting (in the sections to follow) the distinctive meta-theoretical orientation of each of the selected individuals, it needs to be pointed out that they are all physicists with impeccable research credentials, solidly embedded within the rationalist-realist (type II) paradigm common to physics, despite differences between them.

Another way of looking at it is to point out that, despite metaphysical inclinations (for instance, Bohm, Capra and Einstein himself), physics is not primarily a philosophy (general type I); nor poetry (general type III); nor ideology (general type IV). The reason why these particular personages were chosen for discussion is that they more clearly fit the profile of each of the different meta-paradigms introduced here.

Beyond this requirement it is, of course, clear that they do not all share equal status as contributors to physics (Sagan and Capra, despite their own high-level research background, primarily serve as praise-singers, promoters, and also critics, of science in the humanistic tradition). Furthermore, whilst both Einstein and Bohm are acknowledged as path-breaking theoretical physicists, the background of Sagan and Capra is in experimental (applied) physics. But all of them are certainly well known, both within and outside the physics community.

\subsection{David Bohm: The meta-physical physicist (Objectivist-Empyrean)}

Of the four physicists chosen for discussion in this paper David Bohm, whose theory of the Holomovement (with its postulated enfolding and unfolding orders of the universe) which deliberately makes room for a spiritual (non-realist) dimension and the existence of so-called "hidden variables", is clearly the meta-physical or visionary physicist in the objectivist-empyrean (type I) mode of understanding. Einstein (see next section) was also, at times, more transcendentally inclined, even referring to the theoretical physicist as a "tamed meta-physician" (Einstein, 1982/1954). 
Yet, his theory of relativity was based on a thoroughly realist approach to nature and as such did not incorporate or depended on any meta-physical element for its solutions.

Bohm's turning towards a holistic view of the universe came about early with his discovery, as graduate student, in the Lawrence Berkeley Radiation Laboratory that plasma-electrons do not behave as discrete particles but as organised wholes, under certain conditions (Keepin, 1993). This set the theme of his life's work, which was an ongoing investigation of the "Undivided Universe", also the title of his last book which he was proofreading at the time of his death (Olwell, 1999).

For David Bohm the ultimate objects of nature are not discrete elements. Rather, reality consists of a dynamic holistic process in flux, of interconnectedness, which also included thinking about nature. Matter becomes, in his theory, a manifestation of the Explicate Order and conventional science and physics mostly deal with the phenomena of the explicate orders or structures of the universe. Bohm also explicitly acknowledges the role of philosophy in physics: "...all the available interpretations of quantum theory, and indeed of any other physical theory, depend fundamentally on implicit or explicit philosophical assumptions, as well as on assumptions that arise in countless other ways from beyond the field of physics" (Bohm \& Peat, 1987:102).

It must be pointed out that Bohm's holistic theory of the universe is not based on idle speculation but received clear scientific and empirical support. However, the majority of physicists - supporting the Copenhagen school - seem to be of the opinion that Bohm's results do not add anything that was not already achieved or achievable by the purely logical, anti-metaphysical, approaches of quantum mechanics.

In true foundationalist or determinist (type I) fashion, Bohm speaks of an eternal, "super-implicate" order that is ongoing and is pure creation beyond time. Later in his career he explored the possibility of a synthesis of physics and mystical conceptions of reality, meeting with eminent spiritual leaders such as the Dalai Lama and others (see Weber, 1986). He also tried (in the humanist-ideological mode of a type IV knowledge orientation) to establish a movement for those willing to become involved in authentic, non-competitive inter-cultural dialogue with others (Bohm, 1993), the aim of which was to establish a "common consciousness" in order to help save the planet from the excesses of human nature.

\subsection{Albert Einstein: The scientific-explicatory physicist (Objectivist-Empiricist)}

Albert Einstein, by common consent the giant of 20th century science, was the rationalist-realist or objectivist-empiricist (type II) physicist par excellence. His deeply felt need to solve persistent disparities and inconsistencies (such as those concerning the 'ether', and 'absolute space') in the still largely Newtonian physics of the 19th century, formed the backdrop to his revolutionary synthesis (the special and, later, general theories of relativity), which also extended the whole range of modern physics in a way not envisaged since Newton.

A central feature of Einstein's own descriptions of his method and approach is that he consistently juxtaposed and held to be indispensable the physicist's need to address both logical thought (mathematical principles) and experience (experimental observations), both the rational as well as the empirical in establishing the scientific truths about nature (as opposed to an exclusive focus on either one of these).

Yet, being a theoretical physicist, he also strongly emphasized the importance of intuition as that mysterious source of insight into the great principles of nature, which then provided the point of departure for deriving deductive mathematical proofs compatible with empirical phenomena. As he states: "The supreme task of the physicist is to arrive at those universal elementary laws from which the cosmos can be built up by pure deduction. There is no logical path to these laws; only intuition, resting on sympathetic understanding of experience, can reach them...Nobody who has really gone deeply into the matter will deny that in practice the world of phenomena uniquely determines the theoretical system..." (Einstein, 1982/1954: 226). It is also argued that Einstein's own philosophical view of science: "...offers a specialized version of the Kantian synthesis of Empiricism and Rationalism." (Weinert, 2009: 193)

But Einstein, apart from clearly discernible metaphysical leanings (type I), could also on occasion be poetical (type III) about physics, as when he describes the work and life of the physicist as: "The state of mind which enables a man to do work of this kind is akin to that of the religious worshiper or the lover; the daily effort comes from no deliberate intention or program, but straight from the heart" (Einstein, 1982: 227).

Nevertheless, although strongly inclined towards the discovery of the principle laws of nature in the tradition of scientific formalism (logical-mathematical thought), Einstein's work served as trend-setting example of science in the positivist tradition (type II), emphasizing the inseparability of both theory and experience (observation). He expresses it as follows: "We are concerned with the eternal antithesis between the two inseparable components of our knowledge, the empirical and the rational" (Einstein, 1982/1954: 271). 


\subsection{Carl Sagan: The poetical physicist (Subjectivist-Empiricist)}

One way of describing Carl Sagan (an astrophysicist) is as the passionate and strongly poetical apologist for science in the subjectivist-realist (type III) mode of the paradigmatic framework. Not averse to pointing, in a critical manner, to the negative impact of science and technology on humankind, he nevertheless became, via the power of electronic media, known to millions around the world for his charisma and spellbinding stories of scientific achievements and its implications for human life on earth and beyond.

He is the "praise-singer" of physics par excellence. At the same time he and Fritjof Capra were clearly more concerned with the humanistic (subjectivist) question of how we should live than Einstein and to lesser extent Bohm, whose main work addressed the question of knowledge itself. Sagan also did not refrain from highlighting the human implications of scientific discoveries about the cosmos, namely, of the bleakness of humankind's place in the universe. As he states in typical subjectivist, dramatic and emotion-laden fashion: "The tidy anthropocentric proscenium of our ancestors has been replaced by a cold, immense, indifferent Universe in which humans are relegated to obscurity. But I see the emergence in our consciousness of a Universe of magnificence..." (Sagan, 1999: 28).

Sagan actively attempted (in the type IV mode) to educate the public about science and in so doing hoping to curb what he (and others) saw as an alarming degree of gullibility and irrationality (McDonough, 1996). He became a cofounder of a worldwide organisation for the investigation of life on other planets, SETI (Search for Extraterrestrial Intelligence).

In Carl Sagan the physics community had that rare individual who was not only a captivating poet and showman of science (type III), but also achieved eminence as researcher and promoter of science. He was, moreover, a realist and a skeptic concerning spiritual matters; the universe had no soul as far as he was concerned.

\subsection{Fritjof Capra: The missionary physicist (Subjectivist-Empyrean)}

Sagan, with his enthusiasm and inimitable style, essentially wanted to incorporate the world at large in the scientific endeavour, in a manner of speaking. Capra's goal, on the other hand and despite apparent similarities, is to use the knowledge of science and physics as the basis for promoting a holistic and transcendent worldview (type IV) emphasizing mystical values in the Eastern tradition.

Where Sagan may be regarded as a 'lead-singer' in the Church of Reason (read: physics), Capra could be seen as one of its quasi-mystical missionaries; the New Age physicist wishing to realize a deeply spiritual and personal ecological vision for humankind, consonant with the best insights science has to offer.

Taking his lead from Heisenberg's principle of indeterminacy and philosophy of physics, Capra early in his career came to the conclusion that physics, that supposedly most objective of sciences, is a value-laden endeavour; that scientific knowledge in general cannot be separated from values (individually and culturally), and that scientists have both an intellectual and moral responsibility towards humankind (Capra, 1991).

Capra reports that his work sprung from the realization of essential parallels between the worldviews of physicists and mystics. Thus the almost religious fervour with which he over the past twenty-five years spent his time in exploring these parallels, travelling and lecturing all over the world and promoting an ecological vision embedded in the framework of systems theory.

In reading Capra's accounts of how he came to follow his chosen path, it would seem that like many other young people of the sixties and seventies he was deeply influenced by the broadly humanistic cause of the various antiestablishment/ antirational social movements that arose during that period. But in his case, instead of turning him away from science it stimulated him to develop and promote an integrated perspective combining the best of both worlds: science and values, Yang and Yin.

Similar to David Bohm, he also became convinced that the world was a dynamic and interconnected whole, and that the typical positivist and mechanistic paradigm of science merely reinforced an increasingly fragmented existence for humankind, with all its attendant ills, of discrimination, disease, war, political instability, destruction of the ecology, and so on. Convinced that human survival is at stake Capra advocates a radical change in the current scientific worldview, from domination and control of nature to one of harmony, cooperation and non-violence.

To further these ideas he has created, together with others, what he describes as an "ecological think-tank" called the Elmwood Institute. As he concludes in the third edition of the Tao of Physics: "Today we all feel much stronger. We are embedded in the multiple alternative networks of what I have called the "rising culture"-a multitude of movements representing different facets of the same new vision of reality, gradually coalescing to form a powerful force of social transformation" (Capra, 1991: 341). 
In summary, Capra is essentially the missionary-reforming (type IV) physicist, although (perhaps as a result of his scientific training and deep respect for natural science) not as strongly evangelical and obsessed with change, such as is the case with the grand-scale utopianism of other subjectivist-empyrean thinkers like Karl Marx.

\section{In conclusion}

An attempt was made to apply a fundamental framework of basic orientations of mind to the field of physics. A brief sketch of some prominent figures in physics shows that David Bohm represents the type I (metaphysical or objectivistempyrean) tendency; Albert Einstein, the quintessential type II (scientific or objectivist-empiricist) approach; Carl Sagan is primarily the type III (subjectivist-empiricist) figure; whilst Fritjof Capra is the type IV (reforming or subjectivist-empyrean) representative among physicists.

\section{References}

Auyang, S. (1999) A physicist's view of the role of philosophy in science, Physics Today, Vol. 52, (7), p51, 2/3p.

Barnett, S.M. (2000) Scientific revolutions, paradoxes and paradigms, Contemporary Physics, Vol. 41, (3),167-170.

Barrett, J.A. (2000) Philosophical concepts in physics, ISIS: Journal of the History of Science in Society, Vol. 91, (4), 839-841.

Bohm, D. and Peat D. (1987) Science, order, and creativity. New York: Bantam.

Bohm, D. (1993) Science, spirituality, and the present world crisis, ReVision, Vol. 15, (4), 147-153.

Capra, F. (1991) The Tao of Physics, Boston: Shambhala

Easterbrook, G. (2000) The quest for quarks, Wilson Quarterly, Vol. 24, (1), 109-112.

Keepin, W. (1993) River of truth, ReVision, Vol. 16, (1), 32-47.

Einstein, A. (1982/1954) Ideas and opinions, New York: Crown Pubs.

McDonough, T. (1996) In memory of Carl Sagan, Skeptic, Vol. 4, (4), 10-17.

Olwell, R. (1999) Physical isolation and marginalization in physics, ISIS: Journal of the History of Science in Society, Vol. 90, (4), 738757.

Pietersen, H. J. (2011) The Four Types of Knowing -Metaphysical, Scientific, Narrative and Pragmatic: A Meta-Epistemology of Mind, New York: Edwin Mellen Press.

Pietersen, H. J. (2014) The Four Archetypal Orientations of the Mind: Foundational. Experiential, Organizational and Actional, New York: Edwin Mellen Press.

Sagan, C. (1999) Science and technology in the 20th Century: Good and bad, NPQ: New Perspectives Quarterly, Vol. 16, (2), 25-31.

Stenger, V.J. (1993) The myth of quantum consciousness, Humanist, Vol. 53, (3), 13-16.

Weber, R. (1986) Dialogues with scientists and sages, New York: Arkana.

Weinberg, S. (1995) The methods of science...and those by which we live, Academic Questions, Vol. 8, (2), 7-14.

Weinert, F (2009) The modern synthesis: Einstein and Kant. Forum Philosophicum, Vol. 14, 193 - 216. 